\title{
Analysis of the Dynamics of Reasoning Using Multiple Representations ${ }^{1}$
}

\section{Catholijn M. Jonker ${ }^{1}$ and Jan Treur ${ }^{1,2} \quad(\{$ jonker treur $\} @$ cs.vu.nl)}

\author{
${ }^{1}$ Vrije Universiteit Amsterdam, Department of Artificial Intelligence \\ De Boelelaan 1081a, 1081 HV Amsterdam, The Netherlands URL: \\ http://www.cs.vu.nl/ \{jonker, treur\} \\ ${ }^{2}$ Universiteit Utrecht, Department of Philosophy \\ Heidelberglaan 8, 3584 CS Utrecht, The Netherlands
}

\begin{abstract}
This paper presents a formalisation and analysis method for the dynamics of a reasoning process in which multiple representations play a role. Dynamics of reasoning processes are described by reasoning traces consisting of sequences of reasoning states over time. Reasoning states have a compositional structure; they are composed of different parts, for example, for different representations. Transitions between two reasoning states model reasoning steps. In relation to the compositional structure of the states, transitions are classified into a number of types. An example reasoning process involving multiple representations is used to illustrate how its dynamics can be formalised and analysed using the approach.
\end{abstract}

\section{Introduction}

Within Cognitive Science in recent years the dynamical perspective on cognitive phenomena has been emphasized and received much attention. In most literature focussing on the dynamics of cognition, the Dynamical Systems Theory (DST) is taken as a point of departure; e.g., (Port and Gelder, 1995). This theory assumes that, in contrast to the use of symbolic representations, modelling and analysis of dynamics of cognitive phenomena can be done more effectively by using representations based on real numbers and mathematical techniques, in particular difference and differential equations. The convincing examples illustrating the usefulness of this perspective often address lower level cognitive processes such as sensory or motor processing. Indeed one of the advantages of the Dynamical Systems Theory is that it is able to model the temporal aspects of events taking place on a continuous time scale, such as, for example, recognition time, response time, and time involved in motor patterns and locomotion.

\footnotetext{
${ }^{1}$ In: W.D. Gray and C.D. Schunn (eds.), Proceedings of the 24th Annual Conference of the Cognitive Science Society, CogSci 2002. Mahwah, NJ: Lawrence Erlbaum Associates, Inc., 2002, pp. 512-517.
} 
Also some examples of higher level cognitive processes have been addressed using DST; for example the dynamic models for decision making developed by Busemeyer and Townsend (1993). Especially the continuous adaptive aspects of the decision making are covered nicely in this approach. Areas for which the quantitative approach based on DST is assumed to have less to offer are the dynamics of higher level processes with mainly a qualitative character, such as certain capabilities of language processing and reasoning. In the last two decades, within the areas of Computer Science and Artificial Intelligence alternative techniques have been developed to analyse the dynamics of phenomena using qualitative means. Examples are process algebra; transition systems; dynamic and temporal logic; event, situation and fluent calculus; e.g., (Eck, et al. 2001; Hölldobler and Tielscher, 1990; Kowalski and Sergot, 1986; Reiter, 2001). Just as difference or differential equations, these alternative techniques allow to consider and relate states of a process at different points in time. The form in which these relations are expressed can cover both quantitative and non-quantitative aspects. This paper illustrates the usefulness of such an approach for the analysis and formalisation of the dynamics of reasoning. Here a broad perspective is taken on reasoning, subsuming, for example, reasoning involving multiple representations.

A formal analysis method for the dynamics of reasoning is presented and illustrated by an example reasoning pattern involving geometric and arithmetic representations. This pattern is analysed and characterised in terms of a set of dynamic properties. The properties have been formalized, thus enabling automated support of analysis by an analysis environment that has been developed.

Below, first the dynamic perspective on reasoning is discussed in some more detail. Next, the example reasoning pattern is introduced, and the first steps of an analysis are made. Third, a number of dynamic properties identified for the example reasoning pattern are presented. Finally the analysis method is summarised and the contribution of the research presented in the paper is discussed.

\section{Reasoning Dynamics}

Analysis of the cognitive capability to perform reasoning has been addressed from different areas and angles. Within Cognitive Science, the two dominant streams are the syntactic approach (based on inference rules applied to syntactic expressions, as common in logic), e.g., (Rips, 1994), and the semantic approach (based on construction of mental models); e.g., (Johnson-Laird, 1983; Yang and Johnson-Laird, 1999).

Reasoning steps in natural contexts are usually not restricted to the application of logical inference rules. For example, a step in a reasoning process may involve translation of information from one representation form (e.g., geometrical) into another one (e.g., arithmetical). Or, an additional assumption can be made, thus using a dynamic set of premises within the reasoning process. Decisions made at specific points in time during the process, for example, on which representations to use or which assumptions to make, are an inherent part of the reasoning. Such reasoning processes or their outcomes cannot be understood, justified or explained without taking into account these dynamic aspects.

To formalise the dynamics of a reasoning process, traces are used. Reasoning traces are time-indexed sequences of reasoning states over a time frame; for stepwise reasoning processes the set of natural numbers as a time frame is an appropriate choice. The set of all possible reasoning states defines the space where the reasoning takes place. Reasoning traces can be viewed as trajectories in this space, for which every (reasoning) step from one reasoning state to the next one is based on an allowed transition. If the possible reasoning 
states and the allowed reasoning steps or transitions are characterised, the set of proper reasoning traces can be defined as the set of all possible sequences of reasoning states consisting only of allowed transitions.

\section{Reasoning States}

A reasoning state formalises an intermediate state of a reasoning process. The content of such a reasoning state usually can be analysed according to different aspects or dimensions. For example part of the state may contain a geometric representation, another part an arithmetic representation. Accordingly, the reasoning state is structured as a composition of (i.e., a tuple of) a number of parts, indexed by some set I. This index set includes different aspects or views taken on the state, e.g., I = \{geometric, arithmetic $\}$. The set of reasoning states RS can be characterised as a Cartesian product $R S=\Pi_{i \in I} R_{i}$ where $R S_{i}$ is the set of all states for the aspect indicated by i. For example, $\mathrm{RS}_{\text {geometric }}$ may denote the set of all possible geometric representations; note, however, that is also possible to use more dimensions, e.g., different types of geometric representations can be formalised. This Cartesian product formalises the multi-dimensional space where the reasoning takes place. For a reasoning state, which is a vector $S=\left(S_{i}\right) i \in I \in R S$ in this space, the $S_{i}$ are called its components.

\section{Reasoning Steps: Transitions of Reasoning States}

A transition from one reasoning state to another reasoning state, i.e., an element $<\mathrm{S}$, S' $>$ of $\mathrm{RS} \times \mathrm{RS}$, formalises one reasoning step; sometimes also denoted by $\mathrm{S} \rightarrow \mathrm{S}$ '. A reasoning transition relation is a relation on RS $\times$ RS. Such a relation can be used to specify the allowed transitions. Transitions differ in the set of components that are involved. The most complex transitions change all components of the state in one step. However, within stepwise reasoning processes, usually transitions only involve a limited number of components of the state, e.g., only one or two. Transitions can be classified according to which set of components is involved. The most simple types of transition involve a single component transition. Next come transition types where two components are involved. In the current approach we concentrate on these two classes of transition types.

\section{Single component transition types}

For example, when a modification in the reasoning state is made solely within a geometric representation, only the geometric component of the state changes (geometric reasoning step). Or, if a calculation (arithmetic reasoning) step is performed, only the aritmetic component is changing. These single component transitions involve only that component and can be defined within one component only:

$$
\begin{array}{ll}
\text { geometric } & \rightarrow \text { geometric } \\
\text { arithmetic } & \rightarrow \text { arithmetic }
\end{array}
$$

It is also possible that one component of a state is changed by information acquisition, importing information from an external source in the reasoning process.

Transitions involving two components of a reasoning state

Other types of transitions involve more than one component. For example, if information from a geometric representation is translated into an arithmetic form, thereby extending the arithmetic representation, then two components of the state are involved: the arithmetic component and the geometric component. Examples of transition types involving two components are:

geometric $\mathrm{x}$ arithmetic $\rightarrow$ geometric 
(e.g., the geometric representation is extended or modified with results from the arithmetical representation)

arithmetic $\times$ geometric $\rightarrow$ arithmetic

(e.g., the arithmetic representation is extended or modified with results from the geometrical representation)

\section{Reasoning Traces}

Reasoning dynamics results from successive reasoning steps, i.e., successive transitions from one reasoning state to another. Thus a reasoning trace is constructed: a time-indexed sequence of reasoning states $\left(\gamma_{t}\right)_{t \in T}$, where $T$ is the time frame used (the natural numbers). A reasoning trace can be viewed as a trajectory in the multi-dimensional space $R S=\prod_{i \in I} \quad R S_{i}$ of reasoning states. An example of such a reasoning trace will be discussed in Section 3; see also Figure 1. Reasoning traces are sequences of reasoning states subject to the constraint that each pair of successive reasoning states in this trace forms an allowed transition. A trace formalises one specific line of reasoning.

\section{Example Reasoning Process}

An example multi-representation reasoning process is used to illustrate the approach put forward: interaction between arithmetical reasoning and geometrical reasoning. The example focuses on how to determine the outcome of multiplications such as $23 \times 36$. Experiences on using such processes with children (8-9 years old) in class rooms have been reported, e.g., by Dekker et al. (1982), see also (Hutton, 1977). The example can also be extended to an example for children of 13 or 14 years to support algebra by geometric visualisations, e.g., the algebraic identity $(a+b)^{2}=a^{2}+2 a b+b^{2}$ interpreted as the area of a partitioned square of $(a+b) \times(a+b)$ in relation to areas of its parts: a square of $a \times a$, a square of $b \times b$, and two rectangles of $a \times$ b. Also teaching quadratic equations can be supported by such visualisations as discussed, e.g., by Bruner (1968), pp. 59-63. The example pattern shows two types of one component transitions of reasoning states, and two transition types involving two components:

- an arithmetical reasoning step: arithmetic $\rightarrow$ arithmetic

- a geometrical reasoning step: geometric $\rightarrow$ geometric

- a translation of an arithmetical representation into a geometrical representation: geometric $\mathrm{x}$ arithmetic $\rightarrow$ geometric

- a translation of a geometrical representation into an arithmetical representation: arithmetic $x$ geometric $\rightarrow$ arithmetic

The idea is that only simple arithmetical steps are required. The more complicated steps are performed via the geometrical representation. A number of skills are assumed. These skills can be defined in the form of transitions.
A. Assumed arithmetic skills arithmetic $\rightarrow$ arithmetic
aa1. splitting a number in 'tens' and single digits: $28=20+8$
aa2. addition of a list of numbers of up to 4 digits, such as $1200+340+120+6$
aa3. multiplication of two numbers starting with a nonzero digit, followed by zero or more zeros, such as $20 \mathrm{x}$ $8,60 \times 30$.

B. Assumed geometric skills geometric $\rightarrow$ geometric

gg1. partitioning a rectangle in non-overlapping areas based on partitionings of its sides

gg2. determining the area of a figure from the areas of a (non-overlapping) partition 


\section{Assumed translation skills}

geometric $\mathrm{x}$ arithmetic $\rightarrow$ geometric:

ag1. drawing a rectangle with arithmetically given dimensions

ag2. partitioning a line segment according to a splitting of its length

ag3. determining the area of a rectangle from the multiplication of the lengths of its sides

arithmetic $\times$ geometric $\rightarrow$ arithmetic:

ga1. translating the area of a rectangle into the multiplication of the lengths of its sides

ga2. translating the area of a combination of nonoverlapping areas into the sum of the areas

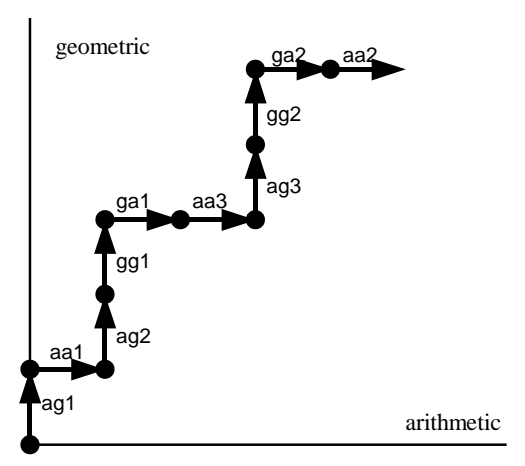

Figure 1: Reasoning trace as a trajectory in a two-dimensional reasoning state space.

The example reasoning trace, based on class room observation (cf. Dekker et al., 1982), forms a trajectory in the two-dimensional reasoning state space

$$
\mathrm{RS}=\mathrm{RS}_{\text {arithmetic }} \times \mathrm{RS}_{\text {geometric }}
$$

This trajectory is depicted in Figure 1. Note that in this Figure only the changing component is visualised by an arrow, not what component affected this change. Therefore, e.g., both a geometric reasoning step and a translation of an arithmetic into a geometric representation are depicted by a vertical arrow. The detailed trace is presented below (next page).

\section{Dynamic Properties}

To specify properties on the dynamics of a reasoning process, the temporal trace language TTL used by Herlea et al. (1999), and Jonker and Treur (1998) is adopted. This is a language in the family of languages to which also situation calculus (Reiter, 2001), event calculus (Kowalski and Sergot, 1986), and fluent calculus (Hölldobler and Tielscher, 1990) belong. In short, in TTL it is possible to express that in a given trace at a certain point in time the reasoning state has a certain (state) property. Moreover, it is possible to relate such state properties at different points in time. As an example, the following (global) property of a reasoning trace $\gamma$ is considered, which expresses that all multiplication problems in two digits eventually will be solved.

\section{GP1}

at any point in time $\mathrm{t}$

if in the reasoning state in trace $\gamma$ at $t$ an arithmetic representation of a multiplication problem for numbers $\mathrm{x}$ and $\mathrm{y}<100$ is present,

then a time point $t \geq t$ exists such that in the reasoning state in $\gamma$ at $t^{\prime}$ an arithmetic representation of a solution $\mathrm{z}$ of this multiplication problem with $\mathrm{z}=\mathrm{x} * \mathrm{y}$ is included. 
The formalisation of this property in TTL is as follows.

$\forall \mathrm{t} \forall \mathrm{x}, \mathrm{y}<100 \operatorname{state}(\gamma, \mathrm{t}$, arithmetic) $\mid==$ multiplication_problem(x,y)

$\Rightarrow \exists \mathrm{t}^{\prime} \geq \mathrm{t} \quad \exists \mathrm{z} \quad \mathrm{z}=\mathrm{x}{ }^{\star} \mathrm{y} \quad$ \&

$\operatorname{state}\left(\gamma, t^{\prime}\right.$, arithmetic) $\mid==$ is_solution_for_multiplication_of $(z, x, y)$

Note that for simplicity no maximal allowed response time has been specified. If desired, this can be simply added by putting a condition $t^{\prime} \leq r$ in the consequent with $r$ the maximal response time.

Starting problem What is the outcome of the multiplication $23 \times 36$ ?

Step 1 ag1 representation translation Create a rectangle of $23 \times 36$.

Step 2 aa1 arithmetic reasoning Split the numbers into the 'tens' and single digits: $23=20+3 ; 36=30+6$

Step 3 ag2 representation translation Translation of the arithmetical splitting of the numbers into partitions of the sides within the geometrical representation.

Step 4 gg1 geometric reasoning Partition the area of the rectangle according to the partitioning of the sides.

Step 5 ga1 representation translation For each part identify the correspond-ing arithmetical expression for its area: $20 \times 30$, $20 \times 6,3 \times 30,3 \times 6$

Step 6 aa3 arithmetic reasoning Determine the outcomes of the four multiplications $20 \times 30=600 ; 20 \times 6=$ $120 ; 3 \times 30=90 ; 3 \times 6=18$

Step 7 ag3 representation translation Identify the areas of the parts of the rectangle based on the outcomes of the multiplications.

Step 8 gg2 geometric reasoning Assert that the area of the rectangle as a whole is the combination of the areas of the parts

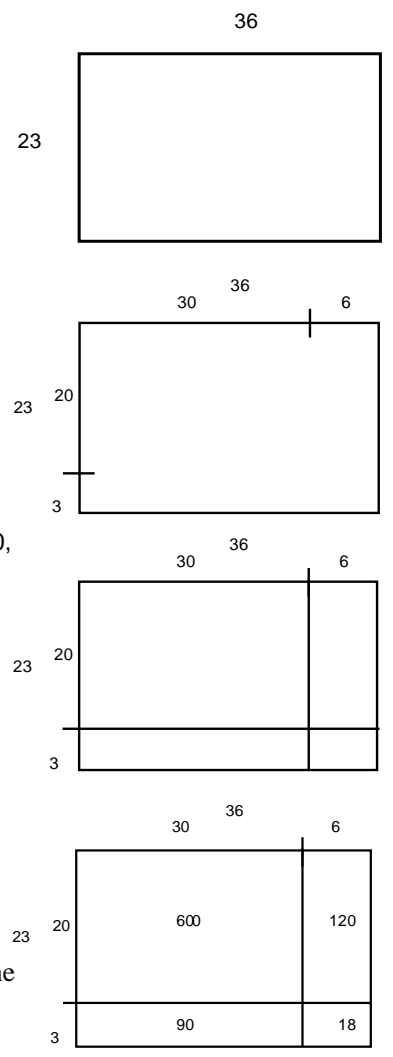

Step 9 ga2 representation translation

Identify the corresponding arithmetical relation: $600+120+90+18$

Step 10 aa2 arithmetic reasoning

Calculate the sum: $600+120+90+18=828$ 


\section{Milestone Properties}

Within the overall reasoning process a number of milestones can be defined, and properties can be identified that express whether the process from one milestone to another one has been performed properly. Apart from the start and the finish, two intermediate milestones were defined: a reasoning state in which the problem has been represented in a geometric representation and it has been decomposed geometrically (after step 4 in the example trace), and a reasoning state in which a geometric representation with numbers in the areas occurs, i.e., in which the subproblems have been solved (after step 7 in the example trace). Accordingly, the following milestone properties have been formulated.

\section{MP1}

at any point in time $t$

if in the reasoning state in trace $\gamma$ at $t$ an arithmetic representation of a multiplication problem for numbers $\mathrm{x}$ and $\mathrm{y}<100$ is present,

then a time point $t \geq t$ exists such that in the reasoning state in $\gamma$ at $t$ ' a geometric representation of a rectangle $A B C D$ is included with points $P$ on $A B$ and $Q$ on $A D$, with $|A B|=x$ and $|A D|=y$

and this rectangle is partitioned into four areas $\mathrm{A}_{11}, \mathrm{~A}_{12}, \mathrm{~A}_{21}, \mathrm{~A}_{22}$ by two lines PP'//AD and QQ'//AB with $\mathrm{P}^{\prime}$ on CD and Q' on BC with $|\mathrm{AP}|=\mathrm{x}_{1},|\mathrm{~PB}|=\mathrm{x}_{2},|\mathrm{AQ}|=\mathrm{y}_{1}$, and $|\mathrm{QD}|=\mathrm{y}_{2}$, where $\mathrm{x}_{1}, \mathrm{y}_{1}$ is the 10-part of $\mathrm{x}$, resp. $\mathrm{y}$, and $\mathrm{x}_{2}, \mathrm{y}_{2}$ is the digit part of $\mathrm{x}$, resp. $\mathrm{y}$.

Here, $|\mathrm{AB}|$ is the length of $\mathrm{AB}$, and // is 'in parallel with'.

\section{MP2}

at any point in time $t$

if in the reasoning state in trace $\gamma$ at $t$ a geometric representation of a rectangle ABCD is included with points $P$ on $A B$ and $Q$ on $A D$, with $|A B|=x$ and $|A D|=y$,

and this rectangle is partitioned into four areas $\mathrm{A}_{11}, \mathrm{~A}_{12}, \mathrm{~A}_{21}, \mathrm{~A}_{22}$ by two lines PP'//AD and QQ'//AB with $\mathrm{P}^{\prime}$ on CD and Q' on BC with $|\mathrm{AP}|=\mathrm{x}_{1},|\mathrm{~PB}|=\mathrm{x}_{2},|\mathrm{AQ}|=\mathrm{y}_{1}$, and $|\mathrm{QD}|=\mathrm{y}_{2}$, where $\mathrm{x}_{1}, \mathrm{y}_{1}$ is the 10-part of $\mathrm{x}$, resp. $\mathrm{y}$, and $\mathrm{x}_{2}, \mathrm{y}_{2}$ is the digit part of $\mathrm{x}$, resp. $\mathrm{y}$,

then a time point $t \geq t$ exists such that in the reasoning state in $\gamma$ at $t$ ' in each of these areas Aij a number zij is represented which equals $\mathrm{x}_{\mathrm{i}} * \mathrm{y}_{\mathrm{j}}$.

\section{MP3}

at any point in time $\mathrm{t}$

if in the reasoning state in trace $\gamma$ at $\mathrm{t}$ a geometric representation of a rectangle ABCD is included with $|\mathrm{AB}|=\mathrm{x}$ and $|\mathrm{AD}|=\mathrm{y}$

and this rectangle is partitioned into four nonoverlapping rectangle areas $A_{11}, A_{12}, A_{21}, A_{22}$,

and in each of these areas $A_{i j}$ a number zij is represented which equals $x_{i^{*}} y_{j}$, where $x=x_{1}+x_{2}$, and $y=y_{1}+$ $\mathrm{y}_{2}$.

then a time point $t \geq t$ exists such that in the reasoning state in $\gamma$ at $t$ ' an arithmetic representation of a solution $\mathrm{z}$ with $\mathrm{z}=\mathrm{x} * \mathrm{y}$ of the multiplication problem $(\mathrm{x}, \mathrm{y})$ is included.

\section{Local Properties}

In this section a number of properties are identified that characterise the reasoning in a more local manner: each property characterises one reasoning step. For the sake of simplicity, for the example reasoning process persistence of representations in reasoning states over time is assumed, so that persistence does not need to be formulated within each of the properties. 


\section{LP1 (arithmetic-geometric)}

at any point in time $\mathrm{t}$

if in the reasoning state in trace $\gamma$ at $\mathrm{t}$ an arithmetic representation of a multiplication problem for numbers $\mathrm{x}$ and $\mathrm{y}<100$ is present,

then a time point $t \geq t$ exists such that in the reasoning state in $\gamma$ at $t$ ' a geometric representation of a rectangle $\mathrm{ABCD}$ with $|\mathrm{AB}|=\mathrm{x}$ and $|\mathrm{AD}|=\mathrm{y}$ is included.

This dynamic property expresses that in reasoning trace $\gamma$, if an arithmetically represented multiplication problem occurs, this eventually is translated into a geometric representation. The formalisation of this property in TTL is as follows.

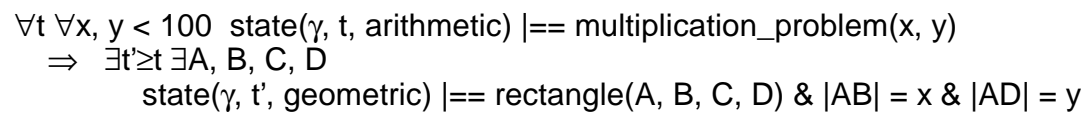

Further local properties are the following (not in any particular order).

\section{LP2 (arithmetic-arithmetic)}

at any point in time $t$

if $\quad$ in the reasoning state in trace $\gamma$ at $\mathrm{t}$ an arithmetic representation of a multiplication problem for numbers $\mathrm{x}$ and $\mathrm{y}<100$ is present,

then a time point $t \geq t$ exists such that in the reasoning state in $\gamma$ at $t$ ' an arithmetic representation of a splitting of the numbers $x$ and $y$ in 'tens' and digits occurs, i.e., $x=x_{1}+x_{2}, y=y_{1}+y_{2}$ with $x_{1}, y_{1}$ multiples of 10 and $\mathrm{x}_{2}, \mathrm{y}_{2}<10$.

\section{LP3 (arithmetic-arithmetic)}

at any point in time $t$

if the reasoning state in trace $\gamma$ at $\mathrm{t}$ contains an arithmetic representation of a multiplication problem for ( $\mathrm{x}$, $\mathrm{y})$, with $\mathrm{x}, \mathrm{y}$ multiple of 10 or less than 10 ,

then a time point $t^{\prime} \geq t$ exists such that in the reasoning state in $\gamma$ at $t^{\prime}$ an arithmetic representation of a solution $\mathrm{z}$ with $\mathrm{z}=\mathrm{x} * \mathrm{y}$ for this multiplication problem for $(\mathrm{x}, \mathrm{y})$ is included.

\section{LP4 (arithmetic-arithmetic)}

at any point in time $\mathrm{t}$

if in the reasoning state in trace $\gamma$ at $\mathrm{t}$ an arithmetic representation of an addition problem for a finite list $\mathrm{z}_{1}$ $, \ldots, z_{n}$ of numbers of up to 4 digits is included,

then a time point $t^{\prime} \geq t$ exists such that in the reasoning state in $\gamma$ at $t^{\prime}$ a solution $\mathrm{z}=\Sigma_{1 \leq \mathrm{i}} \leq \mathrm{n} \mathrm{Z}_{\mathrm{i}}$ of the addition problem is included.

\section{LP5 (arithmetic-geometric)}

at any point in time $t$

if in the reasoning state in trace $\gamma$ at $\mathrm{t}$ an arithmetic representation of a splitting of the numbers $\mathrm{x}$ and $\mathrm{y}$ occurs, i.e., $\mathrm{x}=\mathrm{x}_{1}+\mathrm{x}_{2}, \mathrm{y}=\mathrm{y}_{1}+\mathrm{y}_{2}$

then a time point $t^{\prime} \geq t$ exists such that in the reasoning state in $\gamma$ at $t^{\prime}$ a geometric representation of a rectangle $\mathrm{ABCD}$ with $|\mathrm{AB}|=\mathrm{x}$ and $|\mathrm{AD}|=\mathrm{y}$ is included with points $\mathrm{P}$ on $\mathrm{AB}$ and $\mathrm{Q}$ on $\mathrm{AD}$ such that $|\mathrm{AP}|=\mathrm{x}_{1}$, $|\mathrm{PB}|=\mathrm{x}_{2},|\mathrm{AQ}|=\mathrm{y}_{1}$, and $|\mathrm{QD}|=\mathrm{y}_{2}$.

\section{LP6 (geometric-geometric)}

at any point in time $\mathrm{t}$

if in the reasoning state in trace $\gamma$ at $\mathrm{t}$ a geometric representation of a rectangle ABCD is included with points $\mathrm{P}$ on $\mathrm{AB}$ and $\mathrm{Q}$ on $\mathrm{AD}$,

then a time point $t^{\prime} \geq t$ exists such that in the reasoning state in $\gamma$ at $t^{\prime}$ the rectangle ABCD is partitioned into four areas $\mathrm{A}_{11}, \mathrm{~A}_{12}, \mathrm{~A}_{21}, \mathrm{~A}_{22}$ by two lines $\mathrm{PP}^{\prime} / / \mathrm{AD}$ and $\mathrm{QQ}^{\prime} / / \mathrm{AB}$ with $\mathrm{P}^{\prime}$ on $\mathrm{CD}$ and $\mathrm{Q}^{\prime}$ on $\mathrm{BC}$. 


\section{LP7 (geometric-geometric)}

at any point in time $\mathrm{t}$

if in the reasoning state in trace $\gamma$ at $t$ a geometric representation of a rectangle $A B C D$ is included that is partioned into a number of nonoverlapping areas $A_{1}, \ldots, A_{n}$,

then a time point $t^{\prime} \geq t$ exists such that in the reasoning state in $\gamma$ at $t^{\prime}$ it is asserted that the area of $A B C D$ is the combination of the areas $A_{1}, \ldots, A_{n}$.

\section{LP8 (geometric-arithmetic)}

at any point in time $t$

if in the reasoning state in trace $\gamma$ at $\mathrm{t}$ a geometric representation of a rectangle $\mathrm{ABCD}$ with $|\mathrm{AB}|=\mathrm{x}$ and $|\mathrm{AD}|=\mathrm{y}$ is included with points $\mathrm{P}$ on $\mathrm{AB}$ and $\mathrm{Q}$ on $\mathrm{AD}$ such that $|\mathrm{AP}|=\mathrm{x}_{1},|\mathrm{~PB}|=\mathrm{x}_{2}$, $|\mathrm{AQ}|=\mathrm{y}_{1}$, and $|\mathrm{QD}|=\mathrm{y}_{2}$,

and this rectangle is partioned into four areas $\mathrm{A}_{11}, \mathrm{~A}_{12}, \mathrm{~A}_{21}, \mathrm{~A}_{22}$ by two lines $\mathrm{PP} / / \mathrm{AD}$ and $\mathrm{QQ}^{\prime} / / \mathrm{AB}$ with $\mathrm{P}^{\prime}$ on $\mathrm{CD}$ and $\mathrm{Q}^{\prime}$ on $\mathrm{BC}$,

then a time point $t^{\prime} \geq t$ exists such that in the reasoning state in $\gamma$ at $t^{\prime}$ arithmetic representations of multiplication problems for $\left(\mathrm{x}_{1}, \mathrm{y}_{1}\right),\left(\mathrm{x}_{1}, \mathrm{y}_{2}\right),\left(\mathrm{x}_{2}, \mathrm{y}_{1}\right)$, and $\left(\mathrm{x}_{2}, \mathrm{y}_{2}\right)$ are included.

\section{LP9 (geometric\&arithmetic-geometric)}

at any point in time $t$

if in the reasoning state in trace $\gamma$ at $t$ a geometric representation of a rectangle ABCD is included with points $\mathrm{P}$ on $\mathrm{AB}$ and $\mathrm{Q}$ on $\mathrm{AD}$,

and this rectangle is partioned into four areas $\mathrm{A}_{11}, \mathrm{~A}_{12}, \mathrm{~A}_{21}, \mathrm{~A}_{22}$ by two lines PP'//AD and QQ'//AB with $\mathrm{P}^{\prime}$ on $\mathrm{CD}$ and $\mathrm{Q}^{\prime}$ on $\mathrm{BC}$,

and arithmetic representations of solutions $\mathrm{z}_{11}, \mathrm{z}_{12}, \mathrm{z}_{21}, \mathrm{z}_{22}$ for the multiplication problems for (|AP|, |AQ|), $(|\mathrm{AP}|,|\mathrm{QD}|),(|\mathrm{PB}|,|\mathrm{AQ}|)$, and $(|\mathrm{PB}|,|\mathrm{QD}|)$ are included.

then a time point $t^{\prime} \geq t$ exists such that in the reasoning state in $\gamma$ at $t^{\prime}$ within the geometric representation in each area $\mathrm{A}_{\mathrm{ij}}$, the number $\mathrm{z}_{\mathrm{ij}}$ is represented.

\section{LP10 (geometric-arithmetic)}

at any point in time $\mathrm{t}$

if in the reasoning state in trace $\gamma$ at $\mathrm{t}$ a geometric representation of a rectangle $\mathrm{ABCD}$ is included which is partioned into a number of areas $A_{1}, \ldots, A_{n}$,

and within each of these areas $A_{i}$ a number $z_{i}$ is represented,

then a time point $t^{\prime} \geq t$ exists such that in the reasoning state in $\gamma$ at $t^{\prime}$ an arithmetic representation of an addition problem for $\mathrm{z}_{1}, \ldots, \mathrm{z}_{\mathrm{n}}$ is included.

\section{LP11 (geometric\& arithmetic-arithmetic)}

at any point in time $t$

if in the reasoning state in trace $\gamma$ at $t$ a geometric representation of a rectangle ABCD is included with $|A B|=x$ and $|A D|=y$ that is partitioned into a number of nonoverlapping areas $A_{1}, \ldots, A_{n}$, and within each of these areas $A_{i}$ the number zi is represented,

and an arithmetic representation of a solution $\mathrm{z}$ of the addition problem for $\mathrm{z}_{1}, \ldots, \mathrm{z}_{\mathrm{n}}$ is included,

then a time point $t^{\prime} \geq t$ exists such that in the reasoning state in $\gamma$ at $t^{\prime}$ an arithmetic representation of a solution $\mathrm{z}$ with $\mathrm{z}=\mathrm{x} * \mathrm{y}$ of the multiplication problem $(\mathrm{x}, \mathrm{y})$ is included.

\section{Relationships Between the Dynamic Properties}

A number of logical relationships have been established between the properties above. First of all, the three milestone properties together imply the global property:

$$
\text { MP1 \& MP2 \& MP3 } \Rightarrow \text { GP1 }
$$

Next, each of these milestone properties is implied by a number of local properties:

$$
\begin{array}{rll}
\text { LP1 \& LP2 \& LP5 \& LP6 } & \Rightarrow & \text { MP1 } \\
\text { LP3 \& LP8 \& LP9 } & \Rightarrow & \text { MP2 } \\
\text { LP4 \& LP7 \& LP10 \& LP11 } & \Rightarrow & \text { MP3 }
\end{array}
$$

These logical relationships, which can be depicted as an AND-tree, are helpful in the analysis of errors within a given reasoning trace. First it can be checked whether GP1 holds. If this global property does not hold, the three properties MP1, MP2, MP3 can be checked. Given the logical relationship (0), at least one of them will be found not to hold. This 
pinpoints the cause of the error in part of the process, say MP3. Next, (only) the local properties relating to MP3 are checked, i.e, LP4, LP7, LP10, LP11. Again, due to (3) one of them will be found not to hold. This localises the error.

\section{The Dynamic Analysis Method}

The analysis method for the dynamics of reasoning processes as presented here is summarised as follows.

1. Identify the different dimensions or components of reasoning states.

2. Determine the different types of transitions.

3. Identify relevant dynamic properties for the reasoning

a. for the process as a whole (global properties)

b. for milestones within the process

c. for reasoning steps (local properties)

4. Determine logical relationships between the different dynamic properties, in an ANDtree form; e.g.,

a. local properties imply a milestone property, and

b. milestone properties imply a global property.

5. For a given reasoning trace, check which of the dynamic properties hold and which do not hold. This can take the form of a diagnosis following the tree structure of the relationships between the dynamic properties. A software environment is available to support this checking process.

The dynamic properties identified can be of different types. Some may be assumed to hold for all proper reasoning traces, others may be used to distinguish different types of reasoning traces or reasoners.

\section{Discussion}

The analysis method for the dynamics of reasoning processes put forward and illustrated in this paper was validated on the basis of reports from experiments with 8-9 year old children in classrooms in the Netherlands (Dekker et al., 1982); a similar report has been made by Hutton (1977). This paper shows how an analysis of these dynamics can be made using traces consisting of sequences of reasoning states over time to describe reasoning processes. It is shown for the example reasoning pattern, how characterising dynamic properties can be identified.

The language used to express dynamic allows for precise specification of these dynamic properties, covering both qualitative and quantitative aspects of states and their temporal relations. Moreover, software tools have been developed to (1) support specification of dynamic properties, and (2) automatically check specified dynamic properties against example traces to find out whether the properties hold for the traces. This provides a useful supporting software environment to evaluate empirical data on the dynamics of reasoning processes.

The same analytic method and software tools can also be applied to reasoning traces produced by software simulation models. This applicability supports the comparison of human reasoning with simulated reasoning. 
Further experiments will be conducted, in which also a focus is on the control of the reasoning. For example, at what point in time a translation to a geometric representation is made, and why at that point in time? In the analysis the notion of reasoning strategy will be addressed. Due to the compositional structure of reasoning state a reasoning state can be extended with a component for control information.

Future research will also address the analysis of the dynamics of other types of practical reasoning, both from the syntactical and semantical stream, or their combination; e.g., (Johnson-Laird, 1983; Yang and Johnson-Laird, 1999; Yang and Bringsjord, 2001); see also (Stenning and Lambalgen, 2001). One component of the reasoning state may contain a syntactic formula structure, and another component a mental model or set of mental models. For example, a single component transition can be defined within a syntactic component including $\mathrm{A}$ and $\mathrm{A} \rightarrow \mathrm{B}$, for the derivation of $\mathrm{B}$ (and hence adding it to the component) based on the inference rule modus ponens. Yet another example, within a semantic component is a transition of a set of mental models, thus providing a formalisation of the dynamics of reasoning based on mental models.

\section{References}

Bruner, J.S. (1968). Toward a Theory of Instruction. Norton \& Company, Inc. New York.

Busemeyer, J., and Townsend, J.T. (1993). Decision field theory: a dynamic-cognitive approach to decision making in an uncertain environment. Psychological Review, vol. 100, pp. 432-459.

Dekker, A., Heege, H. ter, and Treffers, A. (1982). Cijferend vermenigvuldigen en delen volgens Wiskobas. Universiteit Utrecht, Freudenthal Institute.

Eck, P.A.T. van, Engelfriet, J., Fensel, D., Harmelen, F. van, Venema, Y. and Willems, M. (2001). A Survey of Languages for Specifying Dynamics: A Knowledge Engineering Perspective. IEEE Transactions on Knowledge and Data Engineering, 13(3):462-496, May/June 2001.

Herlea, D.E., Jonker, C.M., Treur, J., and Wijngaards, N.J.E. (1999). Specification of Behavioural Requirements within Compositional Multi-Agent System Design. In: F.J. Garijo, M. Boman (eds.), Multi-Agent System Engineering, Proc. of the 9th European Workshop on Modelling Autonomous Agents in a Multi-Agent World, MAAMAW'99. Lecture Notes in AI, vol. 1647, Springer Verlag, 1999, pp. 8-27.

Hölldobler, S., and Thielscher, M. (1990). A new deductive approach to planning. New Generation Computing, 8:225-244, 1990.

Hutton, J. (1977). Memoirs of a Maths Teacher 5: Logical Reasoning. In: Mathematics Teaching, vol. 81, pp. 8-12.

Johnson-Laird, P.N. (1983). Mental Models. Cambridge: Cambridge University Press.

Jonker, C.M., and Treur, J. (1998). Compositional Verification of Multi-Agent Systems: a

Formal Analysis of Pro-activeness and Reactiveness. In: W.P. de Roever, H. Langmaack, A. Pnueli (eds.), Proceedings of the International Workshop on Compositionality, COMPOS'97. Lecture Notes in Computer Science, vol. 1536, Springer Verlag, 1998, pp. 350-380. Extended version in: International Journal of Cooperative Information Systems, vol. 11, 2002, pp. 51-92.

Kowalski, R., and Sergot, M. (1986). A logic-based calculus of events. New Generation Computing, 4:67-95, 1986.

Port, R.F., Gelder, T. van (eds.) (1995). Mind as Motion: Explorations in the Dynamics of Cognition. MIT Press, Cambridge, Mass. 
Reiter, R. (2001). Knowledge in Action: Logical Foundations for Specifying and Implementing Dynamical Systems. MIT Press, 2001.

Rips, L.J. (1994). The Psychology of Proof: Deductive reasoning in human thinking. MIT Pres, Cambridge, Mass.

Stenning, K., and Lambalgen, M. van (2001). Semantics as a foundation for Psychology: A Case Study of Wason's Selection Task. Journal of Logic, Language and Information, vol. 10, pp. 273-317.

Yang, Y., and Johnson-Laird, P.N. (1999). A study of complex reasoning: The case GRE 'logical' problems. In M. A. Gernsbacher \& S. J. Derry (Eds.) Proceedings of the Twenty First Annual Conference of the Cognitive Science Society, 767-771.

Yang, Y., and Bringsjord, S. (2001). Mental MetaLogic: a New Paradigm in Psychology of Reasoning. In: L. Chen, Y. Zhuo (eds.), Proc. of the Third International Conference on Cognitive Science, ICCS 2001. Beijing, pp. 199-204. 